\title{
GENERIC TRIVIALIZATIONS OF GEOMETRIC THEORIES
}

\author{
ALEXANDER BERENSTEIN AND EVGUENI VASSILIEV
}

\begin{abstract}
We study the theory $T^{*}$ of the structure induced by parameter free formulas on a "dense" algebraically independent subset of a model of a geometric theory $T$. We show that while being a trivial geometric theory, $T^{*}$ inherits most of the model theoretic complexity of $T$ related to stability, simplicity, rosiness, $N I P$ and $N T P_{2}$. In particular, we show that $T$ is strongly minimal, supersimple of SU-rank 1, or NIP exactly when so is $T^{*}$. We show that if $T$ is superrosy of thorn rank 1 , then so is $T^{*}$, and that the converse holds if $T$ satisfies acl $=$ dcl.
\end{abstract}

\section{INTRODUCTION}

This paper continues the work of the two authors started in [6], where the object of study was the expansion $T^{\text {ind }}$ of a geometric theory $T$ in a language $\mathcal{L}$, obtained by augmenting $\mathcal{L}$ with a predicate for a "dense" algebraically independent subset $H(M)$ of a model $M$ of $T$, thus forming what we referred to as an $H$-structure $(M, H)$. Density here essentially means that $H(M)$ intersects every infinite definable subset of $M$ (one also requires the extension property, see Definition 2.1). Recall that a theory is called geometric, if in all of its models, the algebraic closure satisfies the exchange property, and $T$ eliminates the infinity quantifier $\exists^{\infty}$. The class of geometric theories includes o-minimal, strongly minimal, supersimple SUrank 1 theories, superrosy thorn rank 1 theories (also know as surgical geometric theories), as well as the p-adics in a single sort. In the o-minimal context, the expansion by dense (in the sense of the order) independent subset was introduced by A.Dolich, C. Miller and C. Steinhorn [12]. In [6], we establish basic model theoretic properties of $T^{\text {ind }}$ and show how various stability/simplicity/rosiness properties of $T$ transfer to $T^{i n d}$.

In the present paper we consider the structure induced on $H(M)$ by parameterfree $\mathcal{L}$-formulas, which we denote by $H^{*}(M)$. To any geometric theory $T$ this construction associates a complete theory $T^{*}$ of such structures, the "generic trivialization" of $T$, which itself is a geometric theory with trivial algebraic closure.

To put the study of $T^{*}$ into perspective, we recall from [6] that the notion of an $H$-structure of a geometric theory has a close connection to lovely pairs, another kind of expansion of geometric theories considered in [4] and [5] (and earlier studied in the SU-rank 1 context in [18]; in the o-minimal context in [13]; see also [16] and [3] for stable and simple settings). In fact, one gets a lovely pair from a (sufficiently saturated) $H$-structure if $H(M)$ is replaced with its algebraic closure. It follows from the results in [5] that in the non-trivial weakly one-based (linear) case, the geometry of $M$ modulo $H(M)$ is a disjoint union of projective geometries over division rings. Thus, in the linear case, working modulo $H(M)$ allows one to recover the underlying vector spaces. 
On the other hand, when we restrict to the set $H(M)$, all the information about the pregeometry induced by the algebraic closure in $M$ is lost. Essentially, one looks at the formulas holding on independent tuples in models of $T$. Thus, for example, in the strongly minimal case, $H(M)$ will have no structure other that the one iduced by equality, while in the o-minimal case, one gets a trivial weakly o-minimal expansion of a dense linear order.

However, it turns out that while the first order structure induced on the set $H(M)$ "forgets" the geometry of $T$, it retains most of its "combinatorial" complexity. One can therefore view the construction of an $H$-structure as a way to separate the underlying geometry from the "random noise" (e.g. in the SU-rank 1 case) and/or definable topology (in the o-minimal or C-minimal cases). A natural but challenging question, which is beyond the scope of this paper, is to what extent do the quotient geometry and the generic trivialization describe the original theory $T$ (at least, in the linear case). Our main goal is to investigate how various model theoretic properties of a geometric theory $T$ are reflected in its generic trivialization $T^{*}$.

In Section 2, we establish some preliminary results on $T^{*}$, and show that $T$ is strongly minimal exactly when $T^{*}$ is the theory of equality. We also show that any subset of an $H$-structure $(M, H) \mathcal{L}$-definable over acl $(H(M))$ has a co-finite subset $\mathcal{L}$-definable over $H(M)$, a property that allows us to "pull parameters into $H(M)$ ".

In Section 3, we show that $T$ is $\lambda$-stable, totally transcendental, or supersimple of SU-rank 1 exactly when so is $T^{*}$. We also show that if $T$ is superrosy of thorn rank 1, then so is $T^{*}$, and prove the converse under assumption that $T$ satisfies $\mathrm{acl}=\mathrm{dcl}$. We aslo establish the connection between Morley rank and forking in $T$ and $T^{*}$.

In Section 4, we study the question of NIP and strong dependence. We show that $T$ has NIP (is strongly dependent, dp-minimal) if and only if so is $T^{*}$. We also study the behavior of dp-rank in $T$ and $T^{*}$.

Section 5 is devoted to the study of the $N T P_{2}$ property and burden in $T$ and $T^{*}$.

\section{First properties}

We start this section by recalling basic definitions and results from [6].

Let $T$ be a complete geometric theory in a language $\mathcal{L}$. That is, in any model $M \models T$, the algebraic closure satisfies the Exchange Property and $T$ eliminates the quantifier $\exists^{\infty}$. Let $H$ be a new unary predicate and let $\mathcal{L}_{H}=\mathcal{L} \cup\{H\}$.

Definition 2.1. We say that $(M, H(M))$ is an $H$-structure if

(1) $H(M)$ is an algebraically independent subset of $M$.

(2) (Density/coheir property) If $A \subset M$ is finite dimensional and $q \in S_{1}(A)$ is non-algebraic, there is $a \in H(M)$ such that $a \models q$.

(3) (Extension property) If $A \subset M$ is finite dimensional and $q \in S_{1}(A)$ is non-algebraic, there is $a \in M, a \models q$ and $a \notin \operatorname{acl}(A \cup H(M))$.

It is shown in [6] that $H$-structures exist for any geometric theory $T$. Note that $\operatorname{acl}(H(M))$ is an elementary substructure of $M$ (in fact, $(M, \operatorname{acl}(H(M))$ is a lovely pair in the sense of [4]).

Definition 2.2. Let $A$ be a subset of an $H$-structure $(M, H(M))$. We say that $A$ is $H$-independent if $A$ is algebraically independent from $H(M)$ over $H(A)$. 
Lemma 2.3. Let $(M, H)$ and $(N, H)$ be sufficiently saturated $H$ structures associated to a geometric theory $T$, let $\vec{a} \in M$ and $\vec{a}^{\prime} \in N H$-independent tuples such that $\operatorname{tp}(\vec{a}, H(\vec{a}))=\operatorname{tp}\left(\vec{a}^{\prime}, H\left(\vec{a}^{\prime}\right)\right)$. Then $\operatorname{tp}_{H}(\vec{a})=\operatorname{tp}_{H}\left(\vec{a}^{\prime}\right)$.

In particular the theory of $H$-structures is complete. We write $T^{\text {ind }}$ for this common theory. We normally work with a sufficiently saturated model $(M, H)$ of $T^{\text {ind }}$. Any such model is itself an $H$-structure.

Definition 2.4. Let $(M, H(M))$ be an $H$-structure. Let $\vec{a}=\left(a_{1}, \ldots, a_{n}\right) \in M$. Let $\vec{h} \in H(M)$ be the smallest tuple such that $\vec{a} \downarrow_{\vec{h}} H$. We call $\vec{h}$ the $H$-basis of $\vec{a}$ and we denote it as $H B(\vec{a})$.

Basic properties of the $H$-basis of tuples can be found in [6]. In particular, $H B(\vec{a})$ is a subset of $\operatorname{acl}(\vec{a})$ and is unique up to permutation. In this paper we will need the following result.

Proposition 2.5. Let $A \subset M$ and let $Y \subset H(M)^{n}$ be $\mathcal{L}_{H}$-definable over $A$. Assume that $A=A \cup H B(A)$. Then there is $X \subset M^{n} \mathcal{L}$-definable over $A$ such that $Y=X \cap H(M)^{n}$.

Proof. Let $\vec{a}, \vec{b} \in H(M)^{n}$ be such that $\operatorname{tp}(\vec{a} / A)=\operatorname{tp}(\vec{b} / A)$. Since $A$ is $H$-independent, we get that both $A \vec{a}, A \vec{b}$ are $H$-independent sets and thus by Lemma 2.3 we get $\operatorname{tp}_{H}(\vec{a} / A)=\operatorname{tp}_{H}(\vec{b} / A)$. The result follows by compactness.

In particular, if we take $A=\emptyset$ in the proposition above, then we get that the types of tuples in $H$ are isolated by their $\mathcal{L}$-types.

The following proposition will be used throughout this paper when replacing formulas over $M$ with ones over $H(M)$.

Proposition 2.6. Let $(M, H(M))$ be an $H$-structure, and suppose $D \subset M$ is a set $\mathcal{L}$-definable over $\operatorname{acl}(H(M))$. Then there exists $D^{\prime} \subset M, \mathcal{L}$-definable over $H(M)$, such that $D^{\prime} \subset D$ and $D \backslash D^{\prime}$ is finite.

Proof. Let $D=\phi(M, \vec{a}, \vec{b})$, where $\vec{a} \in H(M)$ and $\vec{b} \in \operatorname{acl}(\vec{a})$, witnessed by an $\mathcal{L}$-formula $\psi(\vec{y}, \vec{a})$. Let $\vec{b}_{0}=\vec{b}, \vec{b}_{1}, \ldots, \vec{b}_{n-1}$ be all the solutions of $\psi(\vec{y}, \vec{a})$. Let $D_{i}=\phi\left(M, \vec{a}, \vec{b}_{i}\right)$. For any nonempty $\sigma \subset n$, let

$$
D_{(\sigma)}=\bigcap_{i \in \sigma} D_{i} \cap \bigcap_{j \in n \backslash \sigma}\left(M \backslash D_{j}\right) .
$$

For each $D_{(\sigma)}$ which is infinite, choose $c_{\sigma} \in H(M) \cap D_{(\sigma)}$ (any infinite $\mathcal{L}$-definable set intersects $H(M)$ ). Then $D_{(\sigma)}$ is $\mathcal{L}$-definable over $\vec{a} c_{\sigma}$. Indeed, it is defined by

$$
\forall \vec{y}\left(\psi(\vec{y}, \vec{a}) \rightarrow\left(\phi(x, \vec{y}, \vec{a}) \leftrightarrow \phi\left(c_{\sigma}, \vec{y}, \vec{a}\right)\right)\right) .
$$

Next, for any $i<n$,

$$
D_{i}=\bigcup_{i \in \sigma, \quad \emptyset \neq \sigma \subset n} D_{(\sigma)}
$$

Let

$$
D_{i}^{\prime}=\bigcup_{i \in \sigma, \quad \emptyset \neq \sigma \subset n, \quad\left|D_{\sigma}\right| \geq \omega} D_{(\sigma)} .
$$

Then $D_{i}^{\prime} \subset D_{i}, D_{i}^{\prime}$ is $\mathcal{L}$-definable over $H(M)$, and $D_{i} \backslash D_{i}^{\prime}$ is finite. In particular, this holds for $D_{0}=D$. 
Remark 2.7. One can easily generalize the above argument to the case when $D \subset$ $M^{n}$ is of dimension $n$. In this setting, there exists $D^{\prime} \subset D$ definable over $H(M)$, with $\operatorname{dim}\left(D \backslash D^{\prime}\right)<n$.

Definition 2.8. We will denote by $H^{*}(M)$ the structure $H(M)$ together with sets $\phi\left(H(M)^{n}\right)$, where $\phi(\vec{x})$ is an $\mathcal{L}$-formula definable in $M$ with no parameters (note that this is the same as the structure induced by $L_{H}$-formulas with no parameters, by Proposition 2.5). More formally, the language of $H^{*}(M)$ consists of the new predicate symbols $R_{\phi}(\vec{x})$ for each such formula $\phi(\vec{x})$, with the obvious interpretation.

Clearly, for any two sufficiently saturated $H$-structures $(M, H)$ and $(N, H)$ of a geometric theory $T$, the structures $H^{*}(M)$ and $H^{*}(N)$ are elementarily equivalent, and are also sufficiently saturated. Thus $T$ gives rise to the unique theory $T^{*}=$ $T h\left(H^{*}(M)\right)$. We will refer to $T^{*}$ as the generic trivalization of $T$.

First, we make some observations about the general model theoretic properties of $H^{*}(M)$.

Remark 2.9. (1) $H^{*}(M)$ is a geometric structure with a trivial (identical) algebraic closure. Elimination of $\exists^{\infty}$ follows from triviality of acl: any formula in one variable with $n$ parameters having more than $n$ realizations is infinite.

(2) For any $\mathcal{L}$-formula $\phi(\vec{x}, y)$ and $\vec{a}=\left(a_{1}, \ldots, a_{n}\right) \in H(M)$, we have

$$
(M, H) \models \exists y(H(y) \wedge \phi(\vec{a}, y)) \Longleftrightarrow M \models \exists^{\infty} y \phi(\vec{a}, y) \vee \bigvee_{i=1}^{n} \phi\left(\vec{a}, a_{i}\right)
$$

Since $T$ is geometric, the latter is an $\mathcal{L}$-formula. This shows that $H^{*}(M)$ has quantifier elimination.

We will now look at the case when the structure of $H^{*}(M)$ is as simple as possible.

Proposition 2.10. $T$ is strongly minimal if and only if $H^{*}(M)$ has no structure (other than the one induced by equality).

Proof. Left to right is clear, since in $T$ there is only one $n$-type of an independent tuple, for any $n$.

Suppose $H^{*}(M)$ has no structure, and $T$ is not strongly minimal. Let $(M, H)$ be an $H$-structure. In $M$ there is an infinite co-infinite definable set $D$. Since $\operatorname{acl}(H(M))$ is an elementary submodel of $M$, we may assume that $D$ is definable over acl $(H(M))$. By Proposition 2.6, there is $D^{\prime} \subset D \mathcal{L}$-definable over $H(M)$, such that $D \backslash D^{\prime}$ is finite. Clearly $D^{\prime} \cap H(M)$ and $H(M) \backslash D^{\prime}$ are disjoint infinite definable subsets of $H^{*}(M)$, a contradiction.

Thus, $T$ is strongly minimal exactly when $T^{*}$ is the theory of equality (which is also equivalent to saying that $T^{*}$ is itself strongly minimal).

Clearly, we cannot expect $T^{*}$ to be as well-behaved in the non-strongly minimal case. For example, if $T$ defines a random graph, then so does $T^{*}$. Moreover, even though $H^{*}(M)$ has a trivial geometry, $T^{*}$ can still interpret some non-trivial structures. In the next example we show how the structure of $M$ can get reflected in $H^{*}(M)$ in a deeper way when $T$ is of Morley rank two.

Example 2.11. Let $T$ be the theory of a vector space over a division ring. Let $T_{P}$ be the theory of lovely (or beautiful, in Poizat's sense) pairs of models of $M$. 
Essentially, $T_{P}$ is the theory of infinite-dimensional pairs of vector spaces. Then $T_{P}$ has Morley rank 2, and the algebraic closures is $T_{P}$ and $T$ coincide (i.e. are given by the linear span), and $T_{P}$ eliminates $\exists^{\infty}$. Thus, $T_{P}$ is a geometric theory with acl $=$ dcl. Consider an $H$-structure $(M, P, H)$ of $T_{P}$. Then $H^{*}(M, P)$ is a linearly independent set having infinite intersection with $P(M)$ and all of its cosets, in particular $H^{*}(M, P)$ has Morley rank two. For $a, b \in H$ write $a E b$ if $a-b \in P . E$ is an $\emptyset$-definable equivalence relation in $H^{*}(M, P)$. We define a product in $H^{*}(M, P) / E$ by $[a]_{E} \cdot[b]_{e}=[c]_{E}$ where $c \in H^{*}(M)$ and $c \in a+b+P$. Then $\left(H^{*}(M) / E, \cdot\right)$ is an interpretable group in $H^{*}(M, P)$ even though the algebraic closure is trivial when restricted to $H^{*}(M, P)$.

Example 2.12. Let $T$ be the theory of the p-adics $\mathbb{Q}_{p}$ in the language $\mathcal{L}_{\text {Div }}$, where $\mathcal{L}$ is the language of rings and for $a, b \in \mathbb{Q}_{p}$ we have that Div $(a, b)$ if and only if $v(a) \leq v(b)$, see [1] for more details. It is well known that $T$ is a geometric theory. Let $\left(\mathbb{Q}_{p}, H\right)$ be a model of $T^{\text {ind }}$. For $a, a^{\prime} \in H^{*}\left(\mathbb{Q}_{p}\right)$ define $E\left(a, a^{\prime}\right)$ if $v(a)=$ $v\left(a^{\prime}\right)$, which is a $\emptyset$-definable equivalence relation. We can define for $a, b \in H^{*}\left(\mathbb{Q}_{p}\right)$, $[a]_{E}+[b]_{E}=[a \cdot b]_{E}$ and $-[a]_{E}=[1 / a]_{E}$. Note that the classes $[a \cdot b]_{E},[1 / a]_{E}$ are realized in $H^{*}\left(\mathbb{Q}_{p}\right)$ by the density property. The group $\left(H^{*}\left(\mathbb{Q}_{p}\right) / E,+\right)$ is an interpretable group in $H^{*}\left(\mathbb{Q}_{p}\right)$ even though the structure $H^{*}\left(\mathbb{Q}_{p}\right)$ is trivial.

Example 2.13. Let $\left(\left(\mathbb{F}_{2}\right)^{\omega},+, 0, P\right)$ be the generic unary predicate expansion of the $\mathbb{F}_{2}$-vector space $\left(\left(\mathbb{F}_{2}\right)^{\omega},+, 0\right)$, in the sense of Chatzidakis-Pillay [10]. Let T be the theory of the structure consisting of $P\left(\left(\mathbb{F}_{2}\right)^{\omega}\right)$ together with the relations $R_{n}\left(x, y_{1}, \ldots, y_{n}\right)$ saying $x+y_{1}+\ldots+y_{n}=0$ (or, equivalently, $\left.x=y_{1}+\ldots+y_{n}\right)$. Note that $T$ is an $\omega$-categorical 1-based SU-rank 1 theory. Let $(M, H)$ be an $H$ structure of $T$. Then the theory $T^{*}$ has quantifier elimination down to formulas of the form $\exists z R_{n}\left(z, x_{1}, \ldots, x_{n}\right)$, and is $\omega$-categorical. Moreover, $T^{*}$ is the model companion of the theory of all structures in the language

$$
\left(P_{2}\left(x_{1}, x_{2}\right), P_{3}\left(x_{1}, x_{2}, x_{3}\right), \ldots\right),
$$

where the relations $P_{n}\left(x_{1}, \ldots, x_{n}\right)$ are symmetric and imply $x_{i} \neq x_{j}$ for all $1 \leq i<$ $j \leq n$. In particular, any random n-hypergraph is definable in $T^{*}$.

Remark 2.14. Note that if $T$ is geometric, then $T^{*}$ is $\omega$-categorical if and only if for any $n T$ has finitely many types of independent $n$-tuples. This is clearly the case when $T$ is strongly minimal, even though $T$ itself may not be $\omega$-categorical.

We will now take a closer look at the relationship between the induced structure on $H(M)$ and the original structure $M$.

Proposition 2.15. Suppose $T$ is a geometric theory, $(M, H)$ a sufficiently saturated $H$-structure. Fix a set $\Gamma$ of $\mathcal{L}$-formulas of the form $\theta(x, \vec{y})$, where $\vec{y}$ can have arbitrary length. Suppose that any definable subset of $H^{*}(M)$ is given by $\theta(x, \vec{b})$ for some $\theta(x, \vec{y})$ in $\Gamma$ and $\vec{b} \in H^{*}(M)$. Then any definable subset of $M$ has a finite symmetric difference with some definable set of the form $\theta(M, \vec{c})$ where $\vec{c} \in M$ and $\theta(x, \vec{y}) \in \Gamma$.

Proof. Suppose a definable set $D$ in $M$ is given by $\phi(x, \vec{c})$. By density property, we may assume that $\vec{c} \in \operatorname{acl}(H(M))$. By Proposition 2.6, changing $D$ to a cofinite subset if needed, we may assume that $\vec{c} \in H(M)$. By the assumption on $\Gamma, \psi(H(M), \vec{c})=\theta(H(M), \vec{b})$ for some $\theta(x, \vec{y}) \in \Gamma$ and $\vec{b} \in H(M)$. Now, if $D=$ $\psi(M, \vec{c})$ has an infinite symmetric difference with $\theta(M, \vec{b})$, by the density property, 
the symmetric difference of $\psi\left(H(M), \vec{c}_{1}\right)$ and $\theta(H(M), \vec{b})$ is also non-empty (in fact, also infinite), a contradiction.

Thus, similarly to strongly minimal or o-minimal structures (where definable sets are described in terms of equality or order), $M$ is " $H$-minimal": if a class of formulas is sufficient for describing definable subsets of $H^{*}(M)$, the same class of formulas will work for $M$.

We finish this section by looking at the case of ordered geometric structures.

Proposition 2.16. Suppose $T$ is a theory of an ordered geometric structure, and $(M, H)$ a sufficiently saturated $H$-structure of $T$. Then

(1) The order restricted to $H(M)$ is dense without endpoints.

(2) If $T$ is a weakly o-minimal expansion of $D L O$ then $H^{*}(M)$ is also weakly o-minimal.

Proof. (1) Follows from the fact that $H^{*}(M)$ has trivial geometry.

(2) Assume $T$ is a weakly o-minimal expansion of DLO. By Proposition 2.5 and Remark 2.9 the definable subsets of $H^{*}(M)$ are the intersection of $\mathcal{L}$-definable subsets of $M$ with $H$. Since the definable subsets of $M$ are finite unions of convex sets and points, the definable subsets of $H^{*}(M)$ are also finite unions of convex sets and points.

Example 2.17. Let $M=\left(\mathbb{Q} \times\{0,1\},<_{\text {lex }}\right)$. Essentially, we replace each element in a dense linear order by a "predecessor-successor" pair. Note that Th(M) is geometric with disintegrated algebraic closure (closure of any element has size 2: the element itself and its successor or predecessor). Note that $M$ is not weakly o-minimal, e.g. the set of all predecessors is dense co-dense in $M$. The structure of $H^{*}(M)$ is that of a dense linear order expanded with a dense co-dense subset: those elements that were predecessors in the original structure $M$. Thus $H^{*}(M)$ is also not weakly o-minimal.

Note that even without the assumption that $M$ is ordered, if $H^{*}(M)$ is an ordered structure, the order must be dense without endpoints. A natural question is: can we extend the order to $M$ ? In other words, does any linear order on $H^{*}(M)$ come from a linear order on $M$ ? The following example shows that it is not the case.

Example 2.18. Let $M=(\mathbb{Q} \times\{0,1\},<)$, where $<$ is the partial order defined by $(x, i)<(y, j)$ if and only if $x<y$. Then in $M$ there is no definable linear order, while $(H(M),<)$ is a dense linear order.

Remark 2.19. Suppose now $T$ is a $C$-minimal theory. Then the structure of $H^{*}(M)$ is weakly $C$-minimal, in the sense that every definable subset of $H^{*}(M)$ is given by a boolean combination of instances of $C$ where the parameters may come from $M$.

\section{Stability, Simplicity AND ROSINESS}

Now we check how generic trivialization behaves with respect to stability, simplicity and rosiness. As we have already shown, $T$ is strongly minimal if and only if so is $T^{*}$ (which is equivalent to $T^{*}$ being the theory of equality).

Proposition 3.1. Let $T$ be a geometric theory. Then $T$ is $\lambda$-stable if and only if $T^{*}$ is $\lambda$-stable. 
Proof. Let $(M, H)$ be a sufficiently saturated $H$-structure of $T$, and $\lambda \geq|T|$. Thus, $T^{*}=T h\left(H^{*}(M)\right)$.

Suppose $T$ is $\lambda$-stable. Let $A \subset H^{*}(M)$ be of size $\leq \lambda$, then there at most $\lambda$ different 1-types over $A$ realized in $M$. Of these types, only the non-algebraic ones together with the family $\{\operatorname{tp}(a / A): a \in A\}$ are realized in $H^{*}(M)$. Thus $T^{*}$ is also $\lambda$-stable.

Suppose $T^{*}$ is $\lambda$-stable.

It suffices to show that for any set $B \subset M$ of size $\leq \lambda$, there at most $\lambda$ different non-algebraic 1-types over $B$ realized in $M$. Next, we may assume that $B=\operatorname{acl}(A)$, where $A \subset H(M)$. By Proposition 2.6, for any $\mathcal{L}$-formula $\phi(x, \vec{a}, \vec{b})$ where $\vec{a} \in A$ and $\vec{b} \in \operatorname{acl}(\vec{a})$, there is $\phi^{\prime}(x, \vec{a}, \vec{c})$ a formula such that $\phi^{\prime}(x, \vec{a}, \vec{c}) \subset \phi(x, \vec{a}, \vec{b})$, they have a finite symmetric difference and the tuple $\vec{c} \in H(M)$. Let $C$ consist of all such $\vec{c}$. Then $|A \cup C| \leq \lambda$. Let $p$ be any non-algebraic 1-type over $B$. Then $p$ is axiomatized by $\mathcal{L}$-formulas of the form $\phi(x, \vec{a}, \vec{b})$, where $\vec{a} \in A$ and $\vec{b} \in \operatorname{acl}(\vec{a})$. Replacing $\phi(x, \vec{a}, \vec{b})$ with $\phi^{\prime}(x, \vec{a}, \vec{c})$, we get a consistent non-algebraic type $p^{\prime}$ over $A \cup C$. Note that if $p_{1} \neq p_{2}$, then $p_{1}^{\prime} \neq p_{2}^{\prime}$. Thus the number of non-algebraic 1 types over $B$ is at most the number of non-algebraic 1-types over $A \cup C$, which is bounded by $\lambda$.

Now we study the special case of totally trascendental theories. Before we start, the reader should notice that if $\varphi(x, \vec{a})$ defines a finite set in $M$, that set may not be realized in $H(M)$. But if $\varphi(x, \vec{a})$ defines an infinite set in $M$, the set has infinitely many realization in $H(M)$. In the next proposition we show that the Morley rank of an infinite formula is the same in $M$ as in $H^{*}(M)$.

Notation 3.2. Let $T$ be a geometric theory, $(M, H)$ a sufficiently saturated $H$ structure of $T$. For any $\mathcal{L}$-formula $\varphi(x, \vec{y})$ and $\vec{a} \in H^{*}(M)$ we write $M R(\varphi(x, \vec{a}))$ for the Morley rank of the formula computed inside $M$ and we write $M R_{H^{*}(M)}(\varphi(x, \vec{a}))$ for the Morley rank of the formula computed inside $H^{*}(M)$.

Proposition 3.3. Let $T$ be a geometric theory, $(M, H)$ a sufficiently saturated $H$-structure of $T$. Then $T$ is totally trascendental if and only if $T^{*}$ is totally trascendental. Moreover for any $\mathcal{L}$-formula $\varphi(x, \vec{y})$ and $\vec{a} \in H^{*}(M)$ if $\varphi(x, \vec{a})$ has infinitely many realizations, $M_{H^{*}(M)}(\varphi(x, \vec{a}))=M R(\varphi(x, \vec{a}))$. In particu$\operatorname{lar} M R(T)=M R\left(T^{*}\right)$

Proof. Claim For any ordinal $\alpha$ and for any formula $\varphi(x, \vec{a})$ where $\vec{a} \in H^{*}(M)$ we have that $M R_{H^{*}(M)}(\varphi(x, \vec{a})) \geq \alpha$ implies $M R(\varphi(x, \vec{a})) \geq \alpha$.

We prove the Claim by induction on $\alpha$. The case $\alpha=0$ and the limit case are clear. If $M R_{H^{*}(M)}(\varphi(x, \vec{a})) \geq 1$ it means that the formula $\varphi(x, \vec{a})$ has infinitely many realizations in $H^{*}(M)$ and thus it has infinitely many realizations in $M$.

Let $\varphi(x, \vec{a})$ be a formula in $H^{*}(M)$ and assume that $M R_{H^{*}(M)}(\varphi(x, \vec{a})) \geq \alpha+1$, with $\alpha \geq 1$. Then there are $\left\{\psi_{i}\left(x, \vec{a}_{i}\right): i \in \mathbb{N}\right\}$ pairwise contradictory formulas in $H^{*}(M)$ which imply $\varphi(x, \vec{a})$ in $H^{*}(M)$ and such that $M R_{H^{*}(M)}\left(\psi_{i}\left(x, \vec{a}_{i}\right)\right) \geq \alpha$ for all $i$. By induction hypothesis $M R\left(\psi_{i}\left(x, \vec{a}_{i}\right)\right) \geq \alpha$. Let $\theta_{i}\left(x, \vec{a}_{i}, \vec{a}\right)=\psi_{i}\left(x, \vec{a}_{i}\right) \wedge$ $\varphi(x, \vec{a})$ and note that $M R\left(\theta_{i}\left(x, \vec{a}, \vec{a}_{i}\right)\right)=M R\left(\psi_{i}\left(x, \vec{a}_{i}\right)\right)$ since the symmetric difference of the two formulas is a finite set in the structure $M$ (they agree in $H^{*}(M)$ ). 
The formulas $\theta_{i}\left(x, \vec{a}, \vec{a}_{i}\right)$ may have finite intersection (which does NOT affect finding Morley ranks since $\alpha \geq 1$ ) and each one implies $\varphi(x, \vec{a})$. It follows that $\operatorname{MR}(\varphi(x, \vec{a})) \geq \alpha+1$.

Claim For any ordinal $\alpha>0$ and $\mathcal{L}$-formula $\varphi(x, \vec{a})$ with $\vec{a} \in H$ if $M R(\varphi(x, \vec{a})) \geq$ $\alpha$ then $M R_{H^{*}(M)}(\varphi(x, \vec{a})) \geq \alpha$.

We prove it by induction on $\alpha$. If $\alpha=1$, it means that $\varphi(x, \vec{a})$ is infinite, then by the density property it intersects $H$ infinitely often and thus $M R_{H^{*}(M)}(\varphi(x, \vec{a})) \geq$ 1. The limit case is clear. Assume the result holds for $\alpha>0$ and that $M R(\varphi(x, \vec{a})) \geq$ $\alpha+1$. Then there are $\psi_{i}\left(x, \vec{b}_{i}\right)$ for $i \in \mathbb{N}$ pairwise disjoint, each of which implies $\varphi(x, \vec{a})$ and such that $M R\left(\psi_{i}\left(x, \vec{b}_{i}\right)\right) \geq \alpha$. We may write $\vec{b}_{i}=\vec{b}_{i}^{0} \vec{b}_{i}^{1}$ so that $\vec{b}_{i}^{0}$ is independent over $\vec{a}, \vec{b}_{<i}$ and $\vec{b}_{i}^{1} \in \operatorname{acl}\left(\vec{a}, \vec{b}_{<i}, \vec{b}_{i}^{0}\right)$. Therefore we can realize $\operatorname{tp}\left(\vec{b}_{i}^{0} / \vec{a}, \vec{b}_{<i}\right)$ inside $H$ and after changing parameters we may assume that $\vec{b}_{i}^{0} \in H$ for every $i$ and that $\vec{b}_{i}^{1} \in \operatorname{acl}\left(\vec{b}_{i}^{0}\right)$. By Proposition 2.6 there are elements $c_{i} \in H$ and formulas $\psi_{i}^{\prime}\left(x, \vec{b}_{i}^{0}, c_{i}\right)$ such that $\psi_{i}^{\prime}\left(x, \vec{b}_{i}^{0}, c_{i}\right)$ defines a cofinite subset of $\psi_{i}\left(x, \vec{b}_{i}\right)$. Note that $\psi_{i}^{\prime}\left(x, \vec{b}_{i}\right)$ for $i \in \mathbb{N}$ are pairwise disjoint and each formula implies $\varphi(x, \vec{a})$. Also since each of $\psi_{i}\left(x, \vec{b}_{i}\right)$ defines an infinite set, $M R\left(\psi_{i}^{\prime}\left(x, \vec{b}_{i}^{0}, c_{i}\right)\right)=$ $M R\left(\psi_{i}\left(x, \vec{b}_{i}\right)\right)$. By induction hypothesis we get that $M R_{H^{*}(M)}\left(\psi_{i}^{\prime}\left(x, \vec{b}_{i}^{0}, c_{i}\right)\right) \geq \alpha$ and so $M R_{H^{*}(M)}(\varphi(x, \vec{a})) \geq \alpha+1$ as we wanted.

Now we turn our attention to the supersimple SU-rank 1 case. Recall that a theory is supersimple of SU-rank 1 exactly when any non-algebraic formula in a single variable does not divide over $\emptyset$.

Proposition 3.4. Let $T$ be a geometric theory. Then $T$ is supersimple of SU-rank 1 if and only if $T^{*}$ is supersimple of SU-rank 1.

Proof. Let $(M, H)$ be a sufficiently saturated $H$-structure of $T$.

Suppose $T$ is supersimple of SU-rank 1. Consider a non-algebraic $\mathcal{L}$-formula $\phi(x, \vec{a})$ where $\vec{a} \in H(M)$. Suppose $\phi(x, \vec{a})$ divides over $\emptyset$ in $H^{*}(M)$, witnessed by an indiscernible sequence $\left(\vec{a}_{i}: i \in \omega\right)$ of tuples in $H(M)$. Thus the partial type $\left\{\phi\left(x, \vec{a}_{i}\right): i \in \omega\right\}$ is not realized in $H(M)$, and hence is algebraic (in $M$ ). Let $e_{1}, \ldots, e_{n}$ be the all its realizations in $M$. We may assume that the sequence $\left(\vec{a}_{i}: i \in \omega\right)$ is indiscernible over $\vec{e}$. Then the sequence $\left(\vec{a}_{i} \vec{e}: i \in \omega\right)$ witnesses that a non-algebraic formula

$$
\phi\left(x, \vec{a}_{0}\right) \wedge \bigwedge_{1 \leq i \leq n} \neg x=e_{i}
$$

divides over $\emptyset$, a contradiction with $T$ being supersimple of SU-rank 1.

Suppose $T^{*}$ is supersimple of SU-rank 1 . Let $\phi(x, \vec{a})$ be a non-algebraic $\mathcal{L}$ formula in $M$, and suppose it divides over $\emptyset$, witnessed by an indiscernible sequence $\left(\vec{a}_{i}: i \in \omega\right)$. Adding a finite acl-independent set $B$, if needed, we may assume that $\left(\vec{a}_{i}: i \in \omega\right)$ is Morley over $B$. Write $\vec{a}_{i}=\vec{a}_{i}^{\prime} \vec{a}_{i}^{\prime \prime}$, where $\vec{a}_{i}^{\prime}$ is acl-independent over $B$, and $\vec{a}_{i}^{\prime \prime} \in \operatorname{acl}\left(\vec{a}_{i}^{\prime} B\right)$. Since the infinite tuple $B \vec{a}_{0}^{\prime} \vec{a}_{1}^{\prime} \ldots$ is acl-independent, we may assume that $B \subset H(M)$ and $\vec{a}_{i}^{\prime} \in H(M)$ for all $i$. By Proposition 2.6, we can find $\vec{c}_{i} \in H(M)$ and an $\mathcal{L}$-formula $\phi^{\prime}(x, \vec{y}, \vec{z})$ such that $\phi^{\prime}\left(M, \vec{a}_{i}^{\prime}, \vec{c}_{i}\right)$ are cofinite subsets of $\phi\left(M, \vec{a}_{i}\right)$. We may also assume that $\left(\vec{a}_{i}^{\prime} \vec{c}_{i}: i \in \omega\right)$ is indiscernible over $B$. Clearly, since $\left\{\phi\left(x, \vec{a}_{i}\right): i \in \omega\right\}$ is inconsistent, so is $\left\{\phi^{\prime}\left(x, \vec{a}_{i}^{\prime}, \vec{c}_{i}\right): i \in \omega\right\}$. Since $\phi^{\prime}\left(H^{*}(M), \vec{a}_{i}^{\prime}, \vec{c}_{i}\right)$ are infinite, this contradicts the assumption that $T^{*}$ is supersimple of SU-rank 1. 
Next, we will consider the case when $T$ is a simple geometric theory, but not necessarily supersimple of SU-rank 1 . Note that in this case, it is still open whether $T^{\text {ind }}$, or at least, $T^{*}$ is also simple. However, we can still say something about the behavior of forking in $T^{*}$.

Proposition 3.5. Let $T$ be a geometric theory, $(M, H)$ a sufficiently saturated $H$ structure of $T$, and suppose $T$ is simple. Let $A \subset B \subset H^{*}(M)$ and let $\vec{c} \in H(M)$ be a tuple. Then if $\operatorname{tp}(\vec{c} / B)$ forks over $A$ (in $M), \operatorname{tp}_{H^{*}(M)}(\vec{c} / B)$ forks over $A$ (in $\left.H^{*}(M)\right)$.

Proof. Assume first that $p(x, B)=\operatorname{tp}(\vec{c} / B)$ forks over $A$ (in $M$ ). Let $\left\{B_{i}: i \in \omega\right\}$ be a $\mathcal{L}$-Morley sequence in $\operatorname{tp}(B / A)$ over $A$ such that $\cup p\left(x, B_{i}\right)$ is inconsistent in $M$. Since the set $B$ is a subset of $H^{*}(M)$, the set $B \backslash A$ is algebraically independent over $A$ and by the density property we may assume that $B_{0} \subset H^{*}(M)$. Since the sequence $\left\{B_{i}: i \in \omega\right\}$ is a $\mathcal{L}$-Morley sequence, the sequence is algebraically independent over $A$, so using an inductive argument we may assume by the density property that $B_{i} \subset H^{*}(M)$ for all $i$. Then $\left\{B_{i}: i \in \omega\right\}$ is an indiscernible sequence over $A$ in $H^{*}(M)$ and $\cup p\left(x, B_{i}\right)$ is inconsistent in $H^{*}(M) \subset M$. This shows that $\operatorname{tp}_{H^{*}(M)}(\vec{c} / B)$ forks over $A$ (in $H^{*}(M)$ ).

Proposition 3.6. Let $T$ be a geometric theory, $(M, H)$ a sufficiently saturated $H$ structure of $T$, and suppose $T$ is simple, that $\mathrm{dcl}=\operatorname{acl}$ in $T$ and that $T^{*}$ is simple. Let $A \subset B \subset H^{*}(M)$ and let $\vec{c} \in H(M)$ be a tuple. Then if $\operatorname{tp}_{H *(M)}(\vec{c} / B)$ forks over $A\left(\right.$ in $\left.H^{*}(M)\right), \operatorname{tp}(\vec{c} / B)$ forks over $A$ (in $M$ ).

Proof. Assume now that $p(\vec{x}, B)=\operatorname{tp}(\vec{c} / B$ ) does not fork over $A$ (in $M$ ). We may write $\vec{c}=\left(c_{1}, \ldots, c_{l}, c_{l+1}, \ldots, c_{n}\right)$, where $c_{1}, \ldots, c_{l}$ are independent over $A$ and $c_{l+1}, \ldots, c_{n} \in \operatorname{dcl}\left(c_{1}, \ldots, c_{l}, A\right)$. Let $f_{l+1}, \ldots, f_{n}$ be definable functions such that $c_{i}=f_{i}\left(c_{1}, \ldots, c_{l}, A\right)$ for $i \geq l+1$. We will prove that $p^{*}(\vec{x}, B)=\operatorname{tp}_{H^{*}(M)}(\vec{c} / B)$ does not fork over $A$ (in $M$ ). Since $T^{*}$ is simple, it suffices to check that for $\left\{B_{i}: i \in \omega\right\}$ a Morley sequence in $\operatorname{tp}_{H^{*}(M)}(B / A)$ over $A$ (in $H^{*}(M)$ ) one has that $\cup_{i \in \omega} p^{*}\left(x, B_{i}\right)$ is consistent in $H^{*}(M)$. By the previous proposition, the sequence $\left\{B_{i}: i \in \omega\right\}$ is a Morley sequence in $\operatorname{tp}(B / A)$ over $A$ (in $M$ ). Since $\operatorname{tp}(\vec{c} / B)$ does not fork over $A$, there is $\vec{d}=\cup_{i \in \omega} p\left(\vec{x}, B_{i}\right)$. Furthermore, we may asssume that $\operatorname{tp}\left(\vec{d} / \cup_{i \in \omega} B_{i}\right)$ does not fork over $A$. In particular, we must have $\operatorname{dim}(\vec{d} / A)=\operatorname{dim}\left(\vec{d} / \cup_{i \in \omega} B_{i}\right)$. Then we can write $\vec{d}=\left(d_{1}, \ldots, d_{l}, d_{l+1}, \ldots, d_{n}\right)$, then $d_{1}, \ldots, d_{l}$ are independent over $\cup_{i \in \omega} B_{i}$ and $d_{l+1}, \ldots, d_{n} \in \operatorname{dcl}\left(d_{1}, \ldots, d_{l}, A\right)$. By the density property we may assume that $d_{1}, \ldots, d_{l} \in H(M)$. Therefore $\operatorname{tp}_{H}\left(c_{1}, \ldots, c_{l}, A\right)=\operatorname{tp}_{H}\left(d_{1}, \ldots, d_{l}, A\right)$, so we must have that

$$
\begin{aligned}
& \operatorname{tp}_{H}\left(c_{1}, \ldots, c_{l}, f_{l+1}\left(c_{1}, \ldots, c_{l}, A\right), \ldots, f_{n}\left(c_{1}, \ldots, c_{l}, A\right), A\right)= \\
& \operatorname{tp}_{H}\left(d_{1}, \ldots, d_{l}, f_{l+1}\left(d_{1}, \ldots, d_{l}, A\right), \ldots, f_{n}\left(d_{1}, \ldots, d_{l}, A\right), A\right),
\end{aligned}
$$

but clearly

$$
f_{l+1}\left(d_{1}, \ldots, d_{l}, A\right), \ldots, f_{n}\left(d_{1}, \ldots, d_{l}, A\right)=\left(d_{l+1}, \ldots, d_{n}\right),
$$

so we get $\operatorname{tp}_{H}(\vec{c}, B)=\operatorname{tp}_{H}\left(\vec{d}, B_{i}\right)$ and thus $\operatorname{tp}_{H^{*}(M)}(\vec{c}, B)=\operatorname{tp}_{H^{*}(M)}\left(\vec{d}, B_{i}\right)$ for all $i$ and $\vec{d} \models \cup_{i \in \omega} p^{*}\left(x, B_{i}\right)$ in $H^{*}(M)$. 
Now we consider the case of thorn rank one theories (or surgical geometric theories). We show that if $T$ is superrosy of thorn rank one then so is its generic trivialization $T^{*}$. We also show the converse under the assumption acl $=\mathrm{dcl}$ in $T$. The proof of the first implication relies on a trick from [2] relating imaginaries in $H^{*}(M)$ with imaginaries in $M$ (in the setting of lovely pairs instead of $H$-structures). We also use the following fact (see [14]):

Fact 3.7. A geometric theory $T$ is superrosy of thorn rank one if and only if for every definable set $X$ (in any number of variables) and a definable equivalence relation $E(x, y)$ on $X$, only finitely many $E$-classes have the same dimension as $X$.

Proposition 3.8. Let $T$ be a geometric theory.

(1) If $T$ is superrosy of thorn-rank 1 then so is $T^{*}$.

(2) If acl $=\mathrm{dcl}$ in $T$, and $T^{*}$ is superrosy of thorn-rank 1, then so it $T$.

Proof. Let $(M, H)$ be a sufficiently saturated $H$-structure of $T$.

(1) Assume that $T$ is superrosy of thorn rank one. Since $T^{*}$ is geometric, by Fact 3.7 it suffices to show that there are no definable subset $S$ of $\left(H^{*}(M)\right)^{m}$ of dimension $n \leq m$ and a definable equivalence relations on $S$ with infinitely many classes of dimension $n$. Note that any such set $S$ is of the form $X \cap H(M)^{m}$, where $X$ is a definable subset of $M^{m}$ (with parameters from $H(M)$ ).

Assume that there is an an $L$-definable set $X \subset M^{m}$ and a $L$-formula $\varepsilon(\vec{x}, \vec{y})$, such that $X \cap H(M)^{m}$ has dimension $n$, and when restricted to $X \cap H(M)^{m}, \varepsilon(-,-)$ defines an equivalence relation with infinitely many classes of dimension $n$. We may assume that $\varepsilon(\vec{a}, \vec{b})$ implies that $\vec{a}, \vec{b} \in X$. Since being superrosy of thorn rank 1 is preserved under reducts and expansions by constants, we may assume that both $X$ and $\varepsilon$ are defined over $\emptyset$.

Case 1: Suppose first $n=m$. Let $X^{\prime}$ consist of all $\vec{a} \in X$ such that $\vec{a}$ is a tuple of distinct elements and there exists another tuple of distinct elements $\vec{b}$ such that $\vec{a}$ and $\vec{b}$ are disjoint (as sets) and $\models \varepsilon(\vec{a}, \vec{b})$. Clearly, $X^{\prime}$ is still $L$-definable over $\emptyset$, has dimension $n$ (in $M$ ), and $\varepsilon$ restricted to $X^{\prime} \cap H(M)^{n}$ is an equivalence relation with infinitely many (in fact, all) classes of dimension $n$.

Now we follow the ideas from [2] and define for $\vec{a}, \vec{b} \in X^{\prime}, E(\vec{a}, \vec{b})="(\varepsilon(\vec{a}, \vec{z}) \vee$ $\varepsilon(\vec{b}, \vec{z})) \wedge \neg(\varepsilon(\vec{a}, \vec{z})) \wedge \varepsilon(\vec{a}, \vec{z}))$ has dimension less than $\mathrm{n} "$. That is, for $\vec{a}, \vec{b} \in X^{\prime}$, $E(\vec{a}, \vec{b})$ holds iff the subset of $X^{\prime}$ defined by $\varepsilon(\vec{a}, \vec{z}) \triangle \varepsilon(\vec{b}, \vec{z})$ has dimension less than $n$. Since $T$ eliminates $\exists^{\infty}$ this relation is definable in $M$. Clearly, it is an equivalence relation (on $X^{\prime}$ ). It remains to show that when restricted to $X^{\prime} \cap H(M)^{n}, E$ coincides with $\varepsilon$, and hence has infinitely many classes of dimension $n$ on $X^{\prime} \cap$ $H(M)^{n}$ and therefore also on $X^{\prime}$ (in the sense of $M$ ).

If $\vec{a}, \vec{b} \in X^{\prime} \cap H(M)^{n}$ and $\models \varepsilon(\vec{a}, \vec{b})$, then clearly $\varepsilon(\vec{a}, \vec{z}) \triangle \varepsilon(\vec{b}, \vec{z})$ is not realized in $H(M)$. Then, by the density property, $\varepsilon(\vec{a}, \vec{z}) \triangle \varepsilon(\vec{b}, \vec{z})$ must have has dimension less than $n$, and thus $=E(\vec{a}, \vec{b})$.

If $\vec{a}, \vec{b} \in X^{\prime} \cap H(M)^{n}$ and $\models \neg \varepsilon(\vec{a}, \vec{b})$, then $\varepsilon(\vec{a}, \vec{z}) \triangle \varepsilon(\vec{b}, \vec{z})$ coincides with $\varepsilon(\vec{a}, \vec{z}) \vee$ $\varepsilon(\vec{b}, \vec{z})$ when restricted to $H(M)$. It follows from the definition of $X^{\prime}$ that each $\varepsilon$ class in $X^{\prime} \cap H(M)^{n}$ has dimension $n$. Hence, $\varepsilon(\vec{a}, \vec{z}) \vee \varepsilon(\vec{b}, \vec{z})$ has dimension $n$, and therefore $\models \neg E(\vec{a}, \vec{b})$.

Case 2: Now suppose $n \leq m$. We will reduce to Case 1. For any function $f:\{1, \ldots, m\} \rightarrow\{1, \ldots, n\}$ consider the function $g_{f}: M^{n} \rightarrow M^{m}$ given by

$$
g_{f}\left(x_{1}, \ldots, x_{n}\right)=\left(x_{f(1)}, \ldots, x_{f(m)}\right) .
$$


Since any $m$-tuple in $X \cap H(M)^{m}$ has at most $n$ distinct entries, we have

$$
X \cap H(M)^{m} \subset \underset{f:\{1, \ldots, m\} \rightarrow\{1, \ldots, n\}}{\bigcup_{f}\left(H(M)^{n}\right) .}
$$

Since there only finitely many such functions $f$, we can find $f:\{1, \ldots, m\} \rightarrow$ $\{1, \ldots, n\}$, such that $\varepsilon(-,-)$ has infinitely many classes of dimension $n$ when restricted to $X \cap g_{f}\left(H(M)^{n}\right)$. Let $Y=g_{f}^{-1}\left(X \cap g_{f}\left(M^{n}\right)\right)$, and for $\vec{h}, \vec{k} \in Y$, let $\varepsilon^{\prime}(\vec{h}, \vec{k})$ denote $\varepsilon\left(g_{f}(\vec{h}), g_{f}(\vec{k})\right)$. Clearly, $Y$ is $L$-definable, has dimension $n$, and $\varepsilon^{\prime}$ is an equivalence relation on $Y \cap H(M)^{n}$. Then apply Case 1 to $Y$ and $\varepsilon^{\prime}$.

(2) Assume now that acl $=\mathrm{dcl}$ in $T, T^{*}$ is superrosy of thorn-rank 1 , but $T$ is not superrosy of thorn-rank 1 . Then there is a definable set $X \subset M^{m}$ of dimension $n \leq m$, and a definable equivalence relation $E$ on $X$ having infinitely many classes of dimension $n$. Since acl $=$ dcl, we may assume that both $X$ and $E$ are definable over $H(M)$. Since being superrosy of thorn rank 1 is preserved under reducts and expansions by constants, we may assume that both $X$ and $E$ are definable over $\emptyset$.

Note that an $E$-class has dimension $n$ exactly when we can find two tuples $\vec{b}$ and $\vec{c}$ that belong to the class, have dimension $n$, realize the same type and are such that $\operatorname{dim}(\vec{b} \vec{c})=2 n$. Let $\vec{x}^{n}=\left(x_{1}, \ldots, x_{n}\right)$ be projection of an $m$-tuple $\vec{x}$ onto first $n$ coordinates. By reordering the variables in necessary, we can find infinitely many $E$ classes of dimension $n$ for which the witnesses have the property that $\operatorname{dim}\left(\vec{b}^{n} \vec{c}^{n}\right)=$ $2 n$ (i.e. the tuples are generated by the first $n$ entries). Fix a sufficiently large cardinal $\kappa$. By compactness, we can construct a sequence $\left(\vec{b}_{\alpha}, \vec{c}_{\alpha}: \alpha<\kappa\right)$ in $X$ such that for any $\alpha<\kappa$ :

$$
\begin{gathered}
\operatorname{tp}\left(\vec{b}_{\alpha}\right)=\operatorname{tp}\left(\vec{c}_{\alpha}\right), \\
=E\left(\vec{b}_{\alpha}, \vec{c}_{\alpha}\right),
\end{gathered}
$$

$\vec{b}_{\alpha}^{n} \vec{c}_{\alpha}^{n}$ is an independent tuple, and for any $\alpha \neq \beta<\kappa, \neg E\left(\vec{b}_{\alpha}, \vec{b}_{\beta}\right)$.

Reducing the sequence, if necessary, we may assume that there is an $\emptyset$-definable function $\vec{f}$ such that

$$
\vec{b}_{\alpha}=\vec{f}\left(\vec{b}_{\alpha}^{n}\right), \quad \vec{c}_{\alpha}=\vec{f}\left(\vec{c}_{\alpha}^{n}\right),
$$

where $\vec{x}^{n}=\left(x_{1}, \ldots, x_{n}\right)$ (projection of an $m$-tuple onto first $n$ coordinates).

Let $X^{H}=\left\{\left(h_{1}, \ldots, h_{n}\right) \in H^{n}: \vec{f}(\vec{h}) \in X, h_{i} \neq h_{j}, i \neq j\right\}$. Define $E^{H}$ on $X^{H}$ by $E^{H}(\vec{h}, \vec{k})$ iff $E(\vec{f}(\vec{h}), \vec{f}(\vec{k}))$. Clearly, both $X^{H}$ and $E^{H}$ are definable in $H^{*}(M)$, and $E^{H}$ is an equivalence relation on $X^{H}$. Moreover, $X^{H}$ has dimension $n$ and the tuples $\vec{b}_{\alpha}^{n}$ and $\vec{c}_{\alpha}^{n}$ witness that $E^{H}$ has infinitely many classes of dimension $n$. Indeed, $\vec{b}_{\alpha}^{n}, \vec{c}_{\alpha}^{n} \in X^{H}, \operatorname{dim}\left(\vec{b}_{\alpha}^{n}, \vec{c}_{\alpha}^{n}\right)=2 n, E^{H}\left(\vec{b}_{\alpha}^{n}, \vec{c}_{\alpha}^{n}\right)$ and $\neg E^{H}\left(\vec{b}_{\alpha}^{n}, \vec{b}_{\beta}^{n}\right)$ for $\alpha \neq \beta$. Contradiction with $T^{*}$ being superrosy of thorn-rank 1 .

In the light of the above results, thorn rank 1 setting seems to be most appropriate for studying generic trivializations. Without this assumption, $H^{*}(M)$ may be "formally" trivial, but become non-trivial when passing to $\left(H^{*}(M)\right)^{e q}$, as in Example 2.11. In fact, if we allow $T$ to have a definable equivalence relation $E$ with infinitely many classes each of which is infinite, any structure definable in $M / E$ will also be definable in $H^{*}(M) / E$. 


\section{NIP AND DP-RANK}

In this section we will study the Independence Property; the setting is the same as before, $T$ is a geometric theory and $(M, H)$ a sufficiently saturated $H$-structure of $T$. Our goal is to prove that $T$ is NIP (strongly dependent) if and only if $T^{*}$ is NIP (strongly dependent) and to study how the dp-rank in both settings are related. The proofs in this section are very close to the ones dealing with NIP in the setting of lovely pairs of geometric structures [7] or just structures expanded with a predicate [9]. We need the following result from [6]:

Fact 4.1. It $T$ is geometric and $T$ has NIP, then $T^{\text {ind }}$ has NIP.

Proposition 4.2. Let $T$ be a geometric theory and suppose $T$ has NIP. Then $T^{*}$ also has NIP.

Proof. Let $(M, H)$ be a sufficiently saturated $H$-structure of $T$. Assume that $T^{*}$ has IP. Then there is $I=\left(\vec{b}_{i}: i \in \omega\right)$ an indiscernible sequence and $a$ in $H^{*}(M)$ such that $\phi\left(a, \vec{b}_{i}\right)$ holds iff $i$ is even. By Remark 2.9 the definable subsets of $H(M)$ are given by $\mathcal{L}$-definable sets intersected with $H(M)$, so there is an $\mathcal{L}$-definable formula $\theta(x, \vec{y})$ such that $\phi(x, \vec{y}) \wedge x \in H(M) \wedge \vec{y} \in H(M)$ is equivalent to $\theta(x, \vec{y}) \wedge x \in$ $H(M) \wedge \vec{y} \in H(M)$.

Note that this sequence $I$ also belongs to $M$, that $a$ belongs to $M$ and that $\theta\left(a, \vec{b}_{i}\right)$ holds iff $i$ is even. So $T^{i n d}$ has the IP and thus by Fact $4.1 T$ has the IP.

Proposition 4.3. Let $T$ be a geometric theory and suppose $T^{*}$ has NIP. Then $T$ has NIP as well.

Proof. Let $(M, H)$ be a sufficiently saturated $H$-structure of $T$.

Suppose $T$ has IP, witnessed by a formula $\phi(x, \vec{y})$ (we may assume that $x$ is a single variable). Thus, in $M$ there exists an indiscernible sequence $I=\left(\vec{b}_{i}: i \in \omega\right)$ and $a$ (non-algebraic over $I$ ) such that $\phi\left(a, \vec{b}_{i}\right)$ holds iff $i$ is even. Extending $I$ we get an indiscernible sequence $J=\left(\vec{b}_{i}: i<\omega+\omega\right)$. Then there exists $a^{\prime}$ (non-algebraic over $J)$ such that $\phi\left(a^{\prime}, \vec{b}_{i}\right)$ holds iff $i=2 n$ or $\omega+2 n$.

Note that the sequence $J=\left(\vec{b}_{i}: \omega \leq i<\omega+\omega\right)$ is independent and indiscernible over $I$. Let $B$ be a finite subset of $I$ such that $\vec{b}_{\omega} \downarrow_{B} I$. Then $\left(\vec{b}_{i}: \omega \leq i<\right.$ $\omega+\omega)$ is independent and indiscernible over $B$. We may assume that $B=\vec{d}$ is an independent tuple. For $\omega \leq i<\omega+\omega$, let $\vec{b}_{i}=\vec{b}_{i}^{0} \vec{b}_{i}^{1}$, where $\vec{b}_{i}^{0}$ is a tuple independent over $\vec{d}$, and $\vec{b}_{i}^{1} \in \operatorname{acl}\left(\vec{d}, \vec{b}_{i}^{0}\right)$. Then the infinite tuple $\vec{d}_{\omega}^{0} \vec{b}_{\omega+1}^{0} \ldots$ is independent over $\emptyset$. We may assume that $a^{\prime}, \vec{d}$, and $\vec{b}_{i}^{0}$ for $\omega \leq i<\omega+\omega$ are all in $H(M)$. After changing parameters, the formula $\phi\left(x, \vec{b}_{i}\right)$ can be written as $\phi\left(x, \vec{d}, \vec{b}_{i}^{0}, \vec{b}_{i}^{1}\right)$ with $\vec{d}, \vec{b}_{i}^{0} \in H$ and $\vec{b}_{i}^{1} \in \operatorname{acl}\left(\vec{d}, \vec{b}_{i}^{0}\right)$ for $\omega \leq i<\omega+\omega$. Note that the way we rewrite the formula does not depend on the index $i$. By Proposition 2.6 and the fact that $J$ is indiscernible there is a sequence $\left\{c_{i}: \omega \leq i<\omega+\omega\right\}$ in $H$ and formulas $\phi^{\prime}\left(x, \vec{d}, \vec{b}_{i}^{0}, c_{i}\right)$ such that $\phi^{\prime}\left(x, \vec{d}, \vec{b}_{i}^{0}, c_{i}\right)$ defines a cofinite subset of $\phi\left(x, \vec{d}, \vec{b}_{i}^{0}, \vec{b}_{i}^{1}\right)$. Furthermore, we may assume that the sequence $\left\{\vec{b}_{i} c_{i}: \omega \leq i<\omega+\omega\right\}$ is indiscernible. Let $J e=\{i=\omega+2 n: n<\omega\}$ and let $J_{o}=\{i=\omega+2 n+1$ : $n<\omega\}$. Since the type $\wedge_{i \in J e} \phi\left(x, \vec{d}, \vec{b}_{i}^{0}, \vec{b}_{i}^{1}\right) \wedge \wedge_{i \in J o} \neg \phi\left(x, \vec{d}, \vec{b}_{i}^{0}, \vec{b}_{i}^{1}\right)$ is not algebraic, so is the type $\wedge_{i \in J e} \phi^{\prime}\left(x, \vec{d}, \vec{b}_{i}^{0}, c_{i}\right) \wedge \wedge_{i \in J o} \neg \phi^{\prime}\left(x, \vec{d}, \vec{b}_{i}^{0}, c_{i}\right)$ and the formula $\left.\phi^{\prime}\left(x, \vec{y}^{0}, z\right)\right)$ witnesses IP for $H^{*}(M)$ and thus for $T^{*}$. 
We will refine our analysis in the setting of strongly dependent theories and compare the $d p$-rank of $T$ and $T^{*}$. Basic facts about dp-rank can be found in [11], more general information about strongly dependent theories can be found in [17]. We only recall the basic definitions :

Definition 4.4. Let $M$ be a sufficiently saturated structure. For a cardinal $\kappa$, an ICT pattern of depth $\kappa$ in variables $\vec{x}$ is a set of formulas $\left\{\varphi_{\alpha}\left(\vec{x} ; \vec{y}^{\alpha}\right): \alpha<\kappa\right\}$ together with an array $\left\{\vec{a}_{n}^{\alpha}: \alpha<\kappa, n<\omega\right\}$ such that $\vec{a}_{n}^{\alpha} \in M_{\vec{y}^{\alpha}}$ and for any $\eta: \kappa \rightarrow \omega$, the type

$$
\bigwedge_{i<\kappa} \varphi_{i}\left(x, \vec{a}_{\eta(i)}^{i}\right) \wedge \bigwedge_{i<\kappa} \bigwedge_{j<\omega, j \neq \eta(i)} \neg \varphi_{i}\left(x, a_{j}^{i}\right)
$$

is consistent. The $d p$-rank for a partial type $p(\vec{x})$ is the maximum cardinal $\kappa$ (possibly finite) such that $p(\vec{x})$ is consistent with an ICT pattern in variables $\vec{x}$ of depth $\kappa$. A theory is strongly dependent if the $d p$-rank of $x=x$ is $\leq \aleph_{0}$.

It is proved in [6] that if $T$ is strongly dependent so is $T^{\text {ind }}$ and vice versa. It is easy to modify the proofs given in Propositions 4.2 and 4.3 to show that $T$ is strongly dependent if and only if $T^{*}$ is strongly dependent. Instead of doing that, we show below how the dp-rank of $x=x$ in $T$ is related to dp-rank of $x=x$ in $T^{*}$.

The reader should note that the dp-rank of $x=x$ can be $\geq n$ for every $n$ but still smaller than $\aleph_{0}$.

Proposition 4.5. Let $T$ be a geometric theory and suppose that $T$ is strongly dependent. Then the $d p$-rank of $x=x$ in $T$ either agrees with the dp-rank of $x=x$ in $T^{*}$ or they differ by one.

Proof. Let $(M, H)$ be a sufficiently saturated $H$-structure of $T$.

Assume that $x=x$ has $d p$-rank greater than or equal to $n$ in $T^{*}$. Then there are $\varphi_{1}\left(x, \vec{y}_{1}\right), \ldots, \varphi_{n}\left(x, \vec{y}_{n}\right) \mathcal{L}$-formulas without parameters and there are sequences $\left\{\left(\vec{a}_{i}^{j}: i<\omega\right): j \leq n\right\}$ that form a ICT pattern of depth $n$ in $H^{*}(M)$. Clearly this is also an ICT pattern of depth $n$ in $M$.

Assume now that $x=x$ has $d p$-rank greater than or equal to $n$ in $T$. Then there exist $\mathcal{L}$ formulas $\varphi_{1}\left(x, \vec{y}_{1}\right), \ldots, \varphi_{n}\left(x, \vec{y}_{n}\right)$ and mutually indiscernible sequences $\left\{\left(\vec{a}_{i}^{j}: i<\omega+\omega\right): j \leq n\right\}$, that form a ICT pattern of depth $n$. Let $I_{1}=\left(\vec{a}_{i}^{1}: i<\right.$ $\omega), \ldots, I_{n}=\left(\vec{a}_{i}^{n}: i<\omega\right)$ and let $J_{1}=\left(\vec{a}_{i}^{1}: \omega \leq i<\omega+\omega\right), \ldots, J_{n}=\left(\vec{a}_{i}^{n}: \omega \leq\right.$ $i<\omega+\omega)$. Note that $J_{1}$ is independent and indiscernible over $I_{1} \cup \cdots \cup I_{n}$. Let $B_{1}$ be a finite subset of $I_{1} \cup \cdots \cup I_{n}$ such that $\vec{a}_{\omega}^{1} \downarrow_{B_{1}} I_{1} \cup \cdots \cup I_{n}$. Then $J_{1}$ is independent and indiscernible over $B_{1}$. In the same way by mutual indiscernability there is $B_{2}$ a finite subset of $I_{1} \cup \cdots \cup I_{n}$ such that $J_{2} \downarrow_{B_{2}} I_{1} \cup J_{1} \cup I_{2} \cdots \cup I_{n}$. And proceeding inductively we can find $B_{n}$ a finite subset of $I_{1} \cup \cdots \cup I_{n}$ such that $J_{n} \downarrow_{B_{n}} I_{1} \cup J_{1} \cup \cdots \cup I_{n-1} \cup J_{n-1} \cup I_{n}$. Let $\vec{b}=B_{1} \cup \cdots \cup B_{n}$, then $J_{1} \cup \cdots \cup J_{n}$ is an independent set over $\vec{b}$. We may assume that $\vec{b}$ is independent. For $\omega \leq i<\omega+\omega$ and $1 \leq j \leq n$ we can write $\vec{a}_{i}^{j}=\vec{a}_{i 1}^{j} \vec{a}_{i 2}^{j}$, where $\vec{a}_{i 1}^{j}$ is a tuple independent over $\vec{b}$, and $\vec{a}_{i 2}^{j} \in \operatorname{acl}\left(\vec{a}_{i 1}^{j} \vec{b}\right)$. Since the elements in $\vec{b} \cup\left\{\vec{a}_{i 1}^{j}: \omega \leq i<\omega+\omega, 1 \leq j \leq n\right\}$ are algebraically independent, we may assume that all the elements in the set belong to $H$. By Proposition 2.6 there is a formula $\varphi_{1}^{\prime}\left(x, \vec{z}_{1}, w\right)$ and there is $c_{i}^{1} \in H$ such that $\varphi_{1}^{\prime}\left(x, \vec{a}_{i 1}^{1}, c_{i}^{1}\right)$ defines a cofinite subset of $\varphi_{1}\left(x, \vec{a}_{i}^{1}\right)$ for $\omega \leq i \leq \omega+\omega$. Repeating the process for the formulas $\varphi_{2}\left(x, \vec{a}_{i}^{2}\right), \ldots, \varphi_{n}\left(x, \vec{a}_{i}^{n}\right)$ we can find formulas 
$\varphi_{2}^{\prime}\left(x, \vec{a}_{i}^{2}, c_{i}^{2}\right), \ldots, \varphi_{n}^{\prime}\left(x, \vec{a}_{i}^{n}, c_{i}^{n}\right)$ which define cofinite subsets of the previous ones and the parameters $c_{i}^{j}$ belong to $H$.

The formulas $\varphi_{1}\left(x, \vec{w}_{1}\right), \ldots, \varphi_{n-1}\left(x, \vec{w}_{n-1}\right)$ together with the sequences $\left\{\left(\vec{a}_{i}^{j}\right.\right.$ : $\omega<i<\omega+\omega): j \leq n-1\}$ form a ICT pattern of depth $n-1$. Note that for each $\eta: n-1 \rightarrow[\omega, \omega+\omega)$, the type

$$
\bigwedge_{i \leq n-1} \varphi_{i}\left(x, \vec{a}_{\eta(i)}^{i}\right) \wedge \bigwedge_{1 \leq i \leq n-1} \bigwedge_{\omega \leq j<\omega+\omega, j \neq \eta(i)} \neg \varphi_{i}\left(x, a_{j}^{i}\right)
$$

has infinitely many realizations (since the pattern can be extended to an ICT pattern of depth $n$ ), in particular it has infinitely many realizations in $H$. Note that exchanging each formula of the form $\varphi_{i}(x)$ for the formula $\varphi_{i}^{\prime}(x)$ only removes a finite number of realizations. Thus the formulas $\varphi_{1}^{\prime}\left(x, \vec{w}_{1}, z\right), \ldots, \varphi_{n-1}^{\prime}\left(x, \vec{w}_{n-1}, z\right)$ together with the sequences $\left\{\left(\vec{a}_{i 1}^{j} c_{i}^{j}: \omega<i<\omega+\omega\right): j \leq n-1\right\}$ form a ICT pattern of depth $n-1$ inside the structure $H^{*}(M)$.

Corollary 4.6. Let $T$ be a geometric theory. Then $T$ is dp-minimal if and only if $T^{*}$ is dp-minimal.

Question 4.7. Are the dp-ranks of $T$ and $T^{*}$ equal?

We end this section by looking at the effect of generic trivialization on VCdimension. The relation in this setting is not clear, since the role of the algebraic closure (as opposed to the complexity of patterns of formulas) takes a more central role.

Remark 4.8. Let $(M, H)$ be an $H$-structure and let $\varphi(\vec{x}, \vec{y})$ be an $\mathcal{L}$-formula. Let $S_{\varphi}=\left\{\varphi\left(M^{m}, \vec{b}\right): \vec{b} \in M^{n}\right\}$, let $S_{\varphi}^{H}=\left\{\varphi\left(H^{m}, \vec{b}\right): \vec{b} \in H^{n}\right\}$ and let $V C(\varphi)=$ $\operatorname{VC}\left(S_{\varphi}\right), V C^{H}(\varphi)=V C\left(S_{\varphi}^{H}\right)$. Then $V C^{H}(\varphi) \leq V C(\varphi)$

Indeed, assume that $A \subset H^{m}$ has size $n$ and that $S_{\varphi}^{H}$ shatters $A$. This means that for every $B \subset A$ there is $\vec{h}_{B} \in H$ such that $B=A \cap \varphi\left(\vec{x}, \vec{h}_{B}\right)$. Then the same witnesses show that $S_{\varphi}$ shatters $A$ in $M$.

\section{NTP 2 AND BURDEN}

In this section we follow the presentation of $N_{T} P_{2}$ theories from [8]. Let $T$ be a complete theory and let $M \models T$ be a sufficiently saturated structure.

Definition 5.1. Let $p(x)$ be a partial type. An inp-pattern in $p(x)$ of depth $\kappa$ consists of $\left(a_{\alpha, i}: \alpha<\kappa, i<\omega\right), \phi_{\alpha}\left(x, y_{\alpha}\right), \alpha<\kappa$ and $k_{\alpha}<\omega$ such that:

(1) $\left\{\left(\phi_{\alpha}\left(x, a_{\alpha, i}\right): i<\omega\right\}\right.$ is $k_{\alpha}$-inconsistent, for each $\alpha<\kappa$

(2) $\left\{\phi_{\alpha}\left(x, a_{\alpha, f(\alpha)}\right): \alpha<\kappa\right\} \cup p(x)$ is consistent, for any $f: \kappa \rightarrow \omega$.

The burden of $p(x)$, denoted $b d n(p)$, is the supremum of the depths of all inppatterns in $p(x)$. If we want to emphasize that we are finding the burden of a type $p(x)$ inside a theory $T$ we write $b d n_{T}(p)$.

Definition 5.2. Let $k<\omega$. A formula $\phi(\vec{x}, \vec{y})$ has $k-T P_{2}$ if there is an array $\left(a_{\alpha, i}: \alpha, i<\omega\right)$ in $M_{\vec{y}}$ such that $\left\{\phi\left(\vec{x}, \vec{a}_{\alpha, i}\right): i<\omega\right\}$ is $k$-inconsistent for every $\alpha<\omega$ and $\left\{\phi\left(\vec{x}, \vec{a}_{\alpha, f(\alpha)}\right): \alpha<\omega\right\}$ is consistent for any $f: \omega \rightarrow \omega$. We say that $\phi(\vec{x}, \vec{y})$ has $T P_{2}$ if it has $k-T P_{2}$ for some $k$. Otherwise we say that $\phi(\vec{x}, \vec{y})$ is $N T P_{2}$, and $T$ is $N T P_{2}$ if every formula is. 
Remark 5.3. Note that if $\phi(\vec{x}, \vec{y})$ has $T P_{2}$ witnessed by the array $\left(\vec{a}_{\alpha, i}: \alpha, i<\omega\right)$, then for for every $f: \omega \rightarrow \omega$ we have that the type $\left\{\phi\left(\vec{x}, \vec{a}_{\alpha, f(\alpha)}\right): \alpha<\omega\right\}$ is not algebraic.

Assume now that $T$ is a geometric theory in a language $\mathcal{L}$ and let $(M, H)$ be a sufficiently saturated $H$-structure. Our goal, as in the previous sections, is to see how the bounds for the burden of types in $T$ relate to bounds on the burden of types in $T^{*}$ and how the failure of $N T P_{2}$ in $T$ relates to the failure of $N T P_{2}$ in $T^{*}$.

We will use the following important facts from $N T P_{2}$ theories:

Fact 5.4. [8] $T$ is $N T P_{2}$ if and only if every formula of the form $\phi(x, \vec{y})$ is $N T P_{2}$, where $x$ is variable in the sort of $M$ (that is, of length one).

Fact 5.5. [15] Assume $T$ has $k-T P_{2}$ witnessed by $\phi(\vec{x} ; \vec{y})$. Then there is an array of parameters $\left\{\vec{a}_{\alpha, i}: \alpha<\omega, i<\omega\right\}$ witnessing $k-T P_{2}$ with $\phi(\vec{x} ; \vec{y})$ such that whenever $i_{0}<i_{1}<\cdots<i_{n}, j_{0}<j_{1}<\cdots<j_{n}$ we have

$$
\begin{gathered}
\operatorname{tp}\left(\vec{a}_{00}, \ldots, \vec{a}_{0 n}, \vec{a}_{10}, \ldots, \vec{a}_{1 n}, \ldots, \vec{a}_{n 0}, \ldots, \vec{a}_{n n}\right)= \\
\operatorname{tp}\left(\vec{a}_{i_{0} j_{0}}, \ldots, \vec{a}_{i_{0} j_{n}}, \vec{a}_{i_{1} j_{0}}, \ldots, \vec{a}_{i_{1} j_{n}}, \ldots, \vec{a}_{i_{n} j_{0}}, \ldots, \vec{a}_{i_{n} j_{n}}\right) .
\end{gathered}
$$

In such a case we say the the sequence of parameters $\left\{\vec{a}_{\alpha, i}: \alpha<\omega, i<\omega\right\}$ is array indiscernible.

Theorem 5.6. Let $T$ be a geometric theory in a language $\mathcal{L}$ and let $(M, H)$ be a sufficiently saturated $H$-structure. If $T$ has $N T P_{2}$, then $T^{*}$ has $N_{T P} P_{2}$.

Proof. Assume that $H^{*}(M)$ has $k-T P_{2}$ for some $k$. By Fact 5.4 and Fact 5.5 there is $\phi(x ; \vec{y})$ and an array indiscernible sequence of parameters $\left\{\vec{a}_{\alpha, i}: \alpha<\omega, i<\omega\right\}$ witnessing $k-T P_{2}$ with $\phi(x ; \vec{y})$ in the structure $H^{*}(M)$. We may assume that the formula $\phi(x ; \vec{y})$ is an $\mathcal{L}$-formula. Since $\left\{\phi\left(x, \vec{a}_{\alpha, i}\right): i<\omega\right\}$ is $k$-inconsistent in $H^{*}(M)$ for every $\alpha<\omega$, the type $\wedge_{i<\omega} \phi\left(\vec{x}, \vec{a}_{\alpha, i}\right)$ is either inconsistent or finite in $M$.

If it is inconsistent, then there is $l \in \mathbb{N}$ such that $\left\{\phi\left(x, \vec{a}_{\alpha, i}\right): i<\omega\right\}$ is $l$ inconsistent and the same formula and the same sequence of parameters witness $l-T P_{2}$ in $M$.

If it is consistent, there is $l \in \mathbb{N}$ such that $\wedge_{i<\omega} \phi\left(x, \vec{a}_{\alpha, i}\right)=\wedge_{i<l} \phi\left(x, \vec{a}_{\alpha, i}\right)$. Let $\left\{e_{1 \alpha}, \ldots, e_{s \alpha}\right\}$ be the set of realizations of the type and let $\vec{e}_{\alpha}=\left(e_{1 \alpha}, \ldots, e_{s \alpha}\right)$. Note that by indiscernability, the value of $l$ and the value of $s$ does not depend on $\alpha$. Let $\psi(x, \vec{y}, \vec{z})=\phi(x, \vec{y}) \wedge_{i<s} x \neq z_{i}$. Then the formula $\psi(x, \vec{y}, \vec{z})$ with the parameters $\left\{\vec{a}_{\alpha, i} \vec{e}_{\alpha}: \alpha<\omega, i<\omega\right\}$ witness $l-T P_{2}$ in $M$.

Theorem 5.7. Let $T$ be a geometric theory in a language $\mathcal{L}$ and let $(M, H)$ be a sufficiently saturated $H$-structure. If the type $x=x$ in $T$ has finite burden so does the type $x=x$ in $T^{*}$ and $b d n_{T}(x=x) \geq b d n_{T^{*}}(x=x) \geq b d n_{T}(x=x)-1$. If the burden of the type $x=x$ in $T$ (resp $T^{*}$ ) is $\kappa$ for some infinite cardinal $\kappa$, then $b d n_{T}(x=x)=b d n_{T^{*}}(x=x)$.

Proof. Assume first that the burden of $x=x$ in $T$ is $n<\omega$. Then there are $\mathcal{L}$ formulas $\phi_{\alpha}(x, \vec{y})$ and there is an array $\left\{a_{\alpha, i}: i<\omega+\omega, 1 \leq \alpha \leq n\right\}$ in $M_{\vec{y}}$ and there are positive integers $\left\{k_{\alpha}: 1 \leq \alpha \leq n\right\}$ such that $\left\{\phi_{\alpha}\left(x, \vec{a}_{\alpha, i}\right): i<\omega+\omega\right\}$ is $k_{\alpha}$-inconsistent and if $f:\{1, \ldots, n\} \rightarrow \omega$ is a function, $\left\{\phi_{\alpha}\left(x, \vec{a}_{\alpha, f(\alpha)}\right): \alpha<k\right\}$ is consistent. We may further assume that each row of the sequence of parameters $\left\{a_{\alpha, i}: i<\omega+\omega, \alpha \leq n\right\}$ is indiscernible over the other rows. 
First we proceed as in the proof of dp-ranks (Proposition 4.5). Let $I_{1}=\left(\vec{a}_{i, 1}\right.$ : $i<\omega), \ldots, I_{n}=\left(\vec{a}_{i, n}: i<\omega\right)$ and let $J_{1}=\left(\vec{a}_{i, 1}: \omega \leq i<\omega+\omega\right), \ldots, J_{n}=\left(\vec{a}_{i, n}:\right.$ $\omega \leq i<\omega+\omega)$. Note that $J_{1}$ is independent and indiscernible over $I_{1} \cup \cdots \cup I_{n}$. Let $B_{1}$ be a finite subset of $I_{1} \cup \cdots \cup I_{n}$ such that $\vec{a}_{\omega, 1} \downarrow_{B_{1}} I_{1} \cup \cdots \cup I_{n}$. Then $J_{1}$ is independent and indiscernible over $B_{1}$. In the same way by mutual indiscernability there is $B_{2}$ a finite subset of $I_{1} \cup \cdots \cup I_{n}$ such that $J_{2} \downarrow_{B_{2}} I_{1} \cup J_{1} \cup I_{2} \cdots \cup I_{n}$. And proceeding inductively we can find $B_{n}$ a finite subset of $I_{1} \cup \cdots \cup I_{n}$ such that $J_{n} \downarrow_{B_{n}} I_{1} \cup J_{1} \cup \cdots \cup I_{n-1} \cup J_{n-1} \cup I_{n}$. Let $\vec{b}=B_{1} \cup \cdots \cup B_{n}$, then $J_{1} \cup \cdots \cup J_{n}$ is an independent set over $\vec{b}$. We may assume that $\vec{b}$ is independent.

For each $\alpha$ and each $i \geq \omega$, we may write $\vec{a}_{\alpha, i}=\vec{a}_{\alpha, i}^{1} \vec{a}_{\alpha, i}^{2}$, where $\vec{a}_{\alpha, i}^{1}$ is independent over $\vec{b}$ and $\vec{a}_{\alpha, i}^{2} \in \operatorname{acl}\left(\vec{a}_{\alpha, i}^{1}, \vec{b}\right)$. We may assume by the density property that $\vec{b} \in H$ and that for each $\alpha$ and $\omega \leq i<\omega+\omega$ we have that $\vec{a}_{\alpha, i}^{1} \in H$. By Proposition 2.6 there is a formula $\phi_{1}^{\prime}\left(x, \vec{b}, \vec{z}_{1}, w\right)$ and there is $c_{1, i} \in H$ such that $\phi_{1}^{\prime}\left(x, \vec{b}, \vec{a}_{1, i}^{1}, c_{1, i}\right)$ defines a cofinite subset of $\phi_{1}\left(x, \vec{a}_{1, i}\right)$ for $\omega \leq i<\omega+\omega$. Similarly for $2 \leq j \leq n$ there are formulas $\phi_{j}^{\prime}\left(x, \vec{b}, \vec{z}_{1}, w\right)$ and there is $c_{j, i} \in H$ such that $\phi_{j}^{\prime}\left(x,, \vec{b}, \vec{a}_{j, i}^{1}, c_{j, i}\right)$ defines a cofinite subset of $\phi_{j}\left(x, \vec{a}_{j, i}\right)$ for $\omega \leq i<\omega+\omega$.

Note that if we consider the array of formulas $\phi_{\alpha}^{\prime}(x, \vec{y})$ and the parameters $\left\{\vec{b}, a_{\alpha, i}^{1} c_{\alpha, i}: \omega<i<\omega+\omega, \alpha<n\right\}$, then $\left\{\phi_{\alpha}^{\prime}\left(x, \vec{b}, \vec{a}_{\alpha, i}^{1} c_{\alpha, i}\right): i<\omega+\omega\right\}$ is $k_{\alpha}$-inconsistent and if $f:\{1, \ldots, n-1\} \rightarrow \omega$ is a function, $\left\{\phi_{\alpha}\left(x, \vec{b}, \vec{a}_{\alpha, f(\alpha)}^{1} c_{\alpha, f(\alpha)}\right)\right.$ : $\alpha<k\}$ has infinitely many solutions and thus it has a solution in $H$. Since all parameters of the array belong to $H$, we have that $b d n_{H^{*}(M)}(x=x) \geq n-1$. If instead of $n$ we have an infinite cardinal the assertion is clear.

Now assume that $b d n_{T^{*}}(x=x) \geq n$. Then there are $\mathcal{L}$-formulas $\phi_{\alpha}(x, \vec{y})$ and there is an array $\left\{a_{\alpha, i}: i<\omega, 1 \leq \alpha \leq n\right\}$ in $H^{*}(M)_{\vec{y}}$ and positive integers $\left\{k_{\alpha}: \alpha \leq n\right\}$ such that $\left\{\phi_{\alpha}\left(x, \vec{a}_{\alpha, i}\right): i<\omega\right\}$ is $k_{\alpha}$-inconsistent in $H^{*}(M)$ and if $f:\{1, \ldots, n\} \rightarrow \omega$ is a function, $\left\{\phi_{\alpha}\left(x, \vec{a}_{\alpha, f(\alpha)}\right): \alpha<k\right\}$ is consistent in $H^{*}(M)$. We may assume that for each $\alpha$, the row $\left\{a_{\alpha, i}: i<\omega\right\}$ is indiscernible. Let $\alpha \leq n$ and consider the type $\wedge_{i \leq \omega} \phi_{\alpha}\left(x, \vec{a}_{\alpha, i}\right)$ in $M$.

If the type is inconsistent, then there is an integer $l_{\alpha}$ such that $\left\{\phi_{\alpha}\left(x, \vec{a}_{\alpha, i}\right): i<\right.$ $\omega\}$ is $l_{\alpha}$-inconsistent in $M$. Let $\psi_{\alpha}(x, \vec{y})=\phi_{\alpha}(x, \vec{y})$ and keep the same parameters $\left\{\vec{a}_{\alpha, i}: i<\omega\right\}$ and let $\vec{e}_{\alpha}=\emptyset$.

If the type is consistent it must be algebraic, and then there is an integer $l_{\alpha}$ such that $\wedge_{i<\omega} \phi_{\alpha}\left(x, \vec{a}_{\alpha, i}\right)=\wedge_{i<l_{\alpha}} \phi_{\alpha}\left(x, \vec{a}_{\alpha, i}\right)$. Let $\vec{e}_{\alpha}=\left\{e_{\alpha 1}, \ldots, e_{\alpha s}\right\}$ be the realizations of the previous type and let $\psi_{\alpha}\left(x, \vec{a}_{\alpha, i}, \vec{e}_{\alpha}\right)=\phi_{\alpha}\left(x, \vec{a}_{\alpha, i}\right) \wedge \wedge_{i \leq s} x \neq e_{i}$. In this case change the $\alpha$ row for $\left\{\psi_{\alpha}\left(x, \vec{a}_{\alpha, i, \vec{e}}\right): i<\omega\right\}$ and change $k_{\alpha}$ for $l_{\alpha}$.

Consider now the pattern asssociated to the formulas $\psi_{\alpha}(x, \vec{y}, \vec{z})$, the array $\left\{\vec{a}_{\alpha, i} \vec{e}_{\alpha}: i<\omega, \alpha \leq n\right\}$ and the integers $\left\{l_{\alpha}: 1 \leq \alpha \leq n\right\}$. If $f:\{1, \ldots, n\} \rightarrow \omega$ is a function, $\left\{\phi_{\alpha}\left(x, \vec{a}_{\alpha, f(\alpha)}\right): \alpha \leq n\right\}$ is consistent in $H^{*}(M)$, so unless the solution coincides with some $e_{\alpha i}$ (there are finitely many of those), we also get a solution for the type $\left\{\psi_{\alpha}\left(x, \vec{a}_{\alpha, f(\alpha)}, \vec{e}_{\alpha}\right): \alpha \leq n\right\}$.

This shows (after removing finitely many rows if necessary) that $b d n_{T^{*}}(x=x) \geq$ $n$.

\section{REFERENCES}

[1] Luc Bélair, Panorama of p-adic model theory, to be published in Ann. Sci. Math. Québec. [2] G Boxall, Lovely pairs and dense pairs of real closed fields, PhD thesis, University of Leeds, 2009. 
[3] I. Ben Yaacov, A. Pillay, E. Vassiliev, Lovely pairs of models, Annals of pure and applied logic, 122 (2003), pp. 235-261.

[4] A. Berenstein and E. Vassiliev, On lovely pairs of geometric structures, Annals of Pure and Applied Logic, Volume 161, Issue 7, April 2010, pp. 866-878.

[5] A. Berenstein and E. Vassiliev, Weakly one-based geometric theories, Journal of Symbolic Logic, Vol.77, No. 2, June 2012.

[6] A. Berenstein and E. Vassiliev, Geometric Theories with a dense independent subset, submitted.

[7] A. Berenstein, A. Dolich and A. Onshuus, The independence property in generalized dense pairs of structures, Journal of Symbolic Logic, 76 (2), 2011, pp. 391-404.

[8] A. Chernikov, Theories without the tree property of the second kind, preprint, available at arXiv:1204.0832.

[9] A. Chernikov and P. Simon, Externally definable sets and dependent pairs, in Israel Journal of Mathematics, 2012, DOI: 10.1007/s11856-012-0061-9.

[10] Z. Chatzidakis and A. Pillay, Generic structures and simple theories, Annals of Pure and Applied Logic, (1998), pp. 71-92.

[11] A. Dolich, J. Goodrick And D. Lippel, Dp-Minimality: Basic Facts and Examples, Notre Dame J. Formal Logic Volume 52, Number 3 (2011), 267-288.

[12] A. Dolich, C. Miller and C. Steinhorn, Expansions of o-minimal structures by independent sets, in preparation.

[13] L. van den Dries, Dense pairs of o-minimal structures, Fund. Math. 157 (1998), pp. 61-78.

[14] J. Gagelman, Stability in geometric theories, Annals of Pure and Applied Logic 132 (2005), 313-326.

[15] B. Kıм, H-J. Kıм, L. Scow, Tree indiscernibilities, revisited, to appear in Archive for Math. Logic.

[16] B. Poizat, Paires de Structures Stables, Journal of Symbolic Logic, 48 (1983), pp 239-249.

[17] S.Shelah, Strongly dependent theories, arXiv:math.LO/0504197, 2005.

[18] E. VAssiliev, Generic pairs of SU-rank 1 structures, Annals of Pure and Applied Logic, 120, (2003), pp. 103-149.

Universidad de los Andes, Cra 1 No 18A-10, Bogotá, Colombia

$U R L$ : www.matematicas.uniandes.edu.co/ aberenst

Grenfell Campus, Memorial Univerity of Newfoundland, Corner Brook, NL A2H 6P9, CANADA

E-mail address: yvasilyev@grenfell.mun.ca

$U R L$ : sites.google.com/site/yevgvas/ 